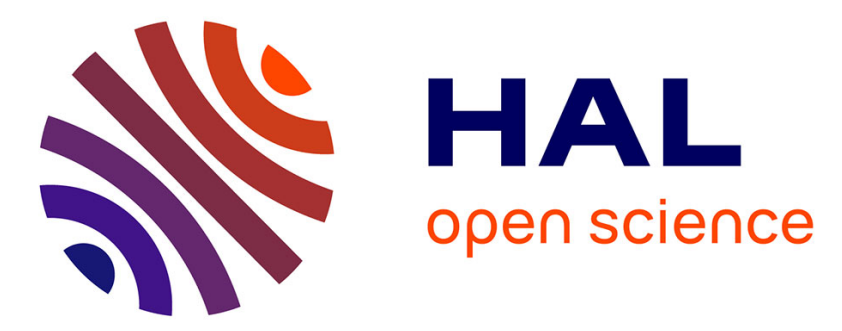

\title{
Partial orthotopic liver transplantation in combination with two-stage hepatectomy: A proof-of-concept explained by mathematical modeling
}

Nicolas Golse, Florian Joly, Quentin Nicolas, Eric Vibert, Pal Dag Line, Irene Vignon-Clementel

\section{To cite this version:}

Nicolas Golse, Florian Joly, Quentin Nicolas, Eric Vibert, Pal Dag Line, et al.. Partial orthotopic liver transplantation in combination with two-stage hepatectomy: A proof-of-concept explained by mathematical modeling. Clinical Biomechanics, 2020, 77, pp.195-200. 10.1016/j.clinbiomech.2020.01.020 . hal-03136651

\section{HAL Id: hal-03136651 \\ https://hal.inria.fr/hal-03136651}

Submitted on 9 Feb 2021

HAL is a multi-disciplinary open access archive for the deposit and dissemination of scientific research documents, whether they are published or not. The documents may come from teaching and research institutions in France or abroad, or from public or private research centers.
L'archive ouverte pluridisciplinaire HAL, est destinée au dépôt et à la diffusion de documents scientifiques de niveau recherche, publiés ou non, émanant des établissements d'enseignement et de recherche français ou étrangers, des laboratoires publics ou privés. 


\section{Partial Orthotopic Liver Transplantation in Combination With Two-stage}

\section{Hepatectomy : a proof-of-concept explained by mathematical modelling}

Nicolas Golse ${ }^{1,2,3}, \mathrm{MD}$, Florian Joly ${ }^{3}, \mathrm{PhD}$, Quentin Nicolas ${ }^{3}$, Eric Vibert ${ }^{1,2}, \mathrm{MD}_{\mathrm{PhD}}$, Pal Dag Line ${ }^{4,5}, \mathrm{MD} \mathrm{PhD}$, Irene Vignon-Clementel ${ }^{3}, \mathrm{PhD}$

${ }^{1}$ Department of Surgery, Hepato-Biliary Centre in Paul-Brousse Hospital, Assistance Publique Hôpitaux de Paris, Villejuif, 94800, France

${ }^{2}$ INSERM, Unit 1193, Villejuif, 94800, France

${ }^{3}$ Inria, centre de recherche de Paris, 2 rue Simone Iff, Paris 75012, France

${ }^{4}$ Department of Transplantation Medicine, Oslo University Hospital, Oslo, Norway

${ }^{5}$ Institute of Clinical Medicine, University of Oslo, Norway

\section{DISCLOSURE}

The authors of this manuscript have no conflicts of interest to disclose.

Category: Brief report

Correspondence and reprint requests:

Dr Nicolas Golse,

Centre Hépato-Biliaire, Hôpital Paul Brousse

12 Avenue Paul Vaillant Couturier

94804 Villejuif Cedex FRANCE

nicolasgolse@me.com 


\section{ABSTRACT}

\section{Background}

Resection And Partial Liver Segment 2/3 Transplantation with Delayed total hepatectomy (RAPID) includes total hepatectomy in 2 steps with small graft transplantation at first stage. To avoid graft portal hyperperfusion, portal vein pressure monitoring is required after revascularization and right portal vein clamping. To date, portal flow modulation has not been reported but simulating hemodynamics in RAPID patients would be useful to anticipate these procedures.

Our team developed hemodynamic OD modeling; we aimed to assess if this mathematical model could be accurately used in the RAPID setting.

\section{Methods}

The modified 0D model was retrospectively tested on 3 patients. We compared our estimated portal vein pressures and portocaval gradients to those intraoperatively measured, as indication to modulate portal flow relies on these measures.

\section{Findings}

Portal pressures measured after right portal vein clamping (end of RAPID procedure) in patients 1,2 and 3 were respectively of 14,16 and $12 \mathrm{mmHg}$ while the simulated pressures were of $13.1,14.8$ and $11.5 \mathrm{mmHg}(\mathrm{p}=0.25)$.

Portocaval gradients measured after right portal vein clamping in the 3 patients were respectively of 10,11 and $7 \mathrm{mmHg}$ while the simulated gradients were of $9.9,11.6$ and 8.3 $\mathrm{mmHg}(\mathrm{p}=0.5)$.

\section{Interpretation}

We succeeded to predict portal vein pressures and portocaval gradients after RAPID. This promising report demonstrates that OD simulation could be a useful tool for human decisionmaking. Moreover, such a patient-specific model could be of importance if we transpose RAPID experience to hepatocellular carcinoma bearing cirrhotics, a population with high probability of portal hypertension after RAPID. 


\section{INTRODUCTION}

The Resection And Partial Liver Segment 2/3 Transplantation with Delayed total hepatectomy (RAPID) concept was recently proposed to increase the availability of grafts for patients with unresectable metastases (1). The RAPID technique includes total hepatectomy (Hx, liver resection) in two steps (Figure 1). First, resection of the segments 1-3 is performed. If needed, an additional resection into segment 4 may be performed. Immediately after resection, the recipient receives a small partial transplant placed orthotopically. Then, portal flow, the main input flow to the liver, is diverted to the graft by selective right portal clamping to facilitate fast graft regeneration. However, to avoid graft portal hyperperfusion or barotrauma, portal vein (PV) pressure monitoring is required after right PV clamping with the objective of maintaining PV pressure below $20 \mathrm{mmHg}$ (2). To date, portal banding, portocaval shunting or splenic artery ligation have not been reported in this setting but could be necessary to reduce liver inflow, particularly in case of pre-existing portal hypertension (PHT) $(1,3)$. As soon as the graft reaches the target volume, usually within 4 weeks posttransplant, the second stage hepatectomy is performed, ending the native diseased liver removal.

For such complex and innovative procedures, numerically simulating hemodynamics in RAPID patients would be useful to anticipate the hemodynamic changes and their needed specific vascular strategies, or to plan the minimal graft size required to avoid small-for-size syndrome. The utility of lumped-models ("0 dimension" electrical models) has already been reported by other teams in liver surgery setting, particularly for simulation of hepatic venous pressure gradient on pathological organs $(4,5)$, hepatic arterial buffer response after LT $(6,7)$ or ex vivo model of liver circulation using machine perfusion devices (8). Recently, our team performed hemodynamic $0 \mathrm{D}$ modeling to simulate major hepatectomies in pigs (9) and understand disease development in cirrhotic rats (10).

To the aim of tailoring the RAPID procedure, we adapted the 0D model in this setting to assess its clinical applicability.

\section{METHODS}

\section{Mathematical model}

A common analogy used in hemodynamics formally identifies fluid flow to an electrical intensity and pressure to voltage, allowing to model the entire circulation with an electronic circuit. Viscous dissipation through vessels and valves is modeled by resistances, and electronic capacitances (or elastances) are used to represent vessels and cardiac compliance. This 0D model - equations do not account for any spatial dimension of the body -, represents the dynamics of the system, driven by the periodic heart ventricular pumps.

The closed-loop model developed in (4) for partial Hx is meant to be simple, yet allowing to represent liver and splanchnic hemodynamics. In addition to the cardio-pulmonary system, it only includes three main blocks: the digestive organs, the liver, and a single block representing all the other organs. The liver is here modeled as two parallel components (2 hemilivers), whose parameters (mainly the resistances of the portal network, arterial network and liver tissue $\mathrm{R}_{\mathrm{PV}}, \mathrm{R}_{\mathrm{HA}}$, and $\mathrm{R}_{\text {liv }}$ ) are indexed on the mass of each hemiliver (Figures 1 and 2 ). The pulsatile activity of the heart is represented by periodic time-varying elastances $E_{i}(t)$ for each of the four cardiac chambers, as well as a nonlinear valve function. Overall, the model leads to a system of 32 differential-algebraic equations, that are solved numerically with the package IDA from Sundials (11).

\section{Parameter tuning procedure (Table 1)}


All the model parameters are tuned with the heart rate (HR) and hemiliver masses to match six of a patient's major hemodynamic variables preoperatively: cardiac output (estimated from thermodilution technique or pulse contour analysis from arterial catheter flow data), mean arterial pressure (measured from arterial catheter), vena cava pressure (central venous catheter), portal pressure ( $\mathrm{P}_{\mathrm{PV}}$, measured from direct portal vein puncture with $25 \mathrm{G}$ needle), portal flow $\left(\mathrm{QPV}_{\mathrm{PV}}\right)$ and hepatic arterial flow $\left(\mathrm{Q}_{\mathrm{HA}}\right.$, flow measures from transit time ultrasound flowmeter positioned around the studied vessel). The digestive organs, other organs and liver resistances are first computed according to these values, as detailed in (4). Capacitances are fixed for the lungs, digestive organs and other organs among all patients (12). The initial resistance values of each hemiliver are taken inversely proportional to the native lobe mass while the capacitances are chosen proportional to the native lobe masses. The last parameters to be estimated are the amplitude and baseline elastance parameters ( 2 per chamber, (3)). They are estimated based on an efficient optimization algorithm (covariance matrix adaptation evolution strategy [CMA-ES]) until they approach the above-detailed hemodynamic variables best (L2 score -representing weighted average differences between measured and simulated data- being as low as possible). Briefly, CMA-ES uses a stochastic, derivative-free method for numerical optimization; it searches best parameter sets based on the principle of biological evolution (individuals created at each iteration/generation from parents; hundreds of iterations required for each simulation) (13).

\section{Model of RAPID sequence}

The RAPID sequence was modeled as follows, with timings between steps chosen to reach a stabilized system (Figure 1):

1/ First hepatectomy: the left lobe resection is simulated by decreasing its mass to virtually 0 , which has the effect of increasing left PV, left HA and native left lobe resistances to an infinite value, mimicking vessel clamping.

2/ Transplantation: for LT simulation, the graft resistances replace the native left lobe resistances. We needed an estimation of these graft resistances: we assumed that the intrinsic resistance of any healthy liver part is constant since no fibrotic or cirrhotic livers were included in this work (i.e. $R_{H A} \times M, R_{P V} \times M$ and $R_{\text {liv }} \times M$ are constant, where $R_{x}$ are the resistances and $\mathrm{M}$ is the mass). This assumption relies on the fact that the resistance of $\mathrm{N}$ parallel branches of a hydraulic circuit is inversely proportional to the number of branches and that a larger liver has more small vessels. We averaged these values over our database from Paul Brousse Hospital with healthy livers (experiments not shown).

3/ Right PV clamping is simulated by increase of right PV resistance to infinity.

4/ Second hepatectomy (right side): the right HA and native right lobe resistances are increased to infinite values. This stage was not modeled because the primary endpoint (clinically relevant) was the portal pressure after PV clamping only. The removal of the right liver only requires HA division (PV already occluded), which does not increase significantly the PV pressure.

\section{Clinical application}

The 0D model was retrospectively tested on 3 datasets of RAPID patients with the following provided data: native right / left liver weights (from liver CT-scan volumetries and assuming that liver density is 1), percentage of left liver resected, initial (= pre-resection) portal / central (inferior vena cava) / mean arterial pressures, graft liver weight. In order to calibrate the model resistances and heart elastances, we lacked four parameters from the actual patients: HR, cardiac output (CO), QPV and $\mathrm{Q}_{\mathrm{HA}}$. Because the patients were not cirrhotic and presented normal body surface indexes, we assumed that $\mathrm{HR}=70 \mathrm{BPM}, \mathrm{CO}=5 \mathrm{~L} / \mathrm{min}$, and that $\mathrm{PV}$ and $\mathrm{HA}$ flows represented respectively $20 \%$ and $5 \%$ of CO (14). 
After calibration to patient's data, we compared our estimated PPV and portocaval gradients (PCG) to those intraoperatively measured with a nonparametric paired test (Wilcoxon), as indication to modulate portal flow mainly relies on these measures. We also assessed the correlation performance with the Spearman test.

\section{RESULTS}

Calibration provided satisfactory low L2 scores $<0.06$ (see Table 1 for more details). After successful calibration to pre-resection data, the different steps were simulated (Figure 3).

Regarding $\mathrm{P}_{\mathrm{PV}}$ evolution, the complete simulation resulted in successive slight increase after Hx $(+11 \%[10 ; 12]$ median increase), followed by decrease after LT $(-4.6 \%[-3.7 ;-4.7])$ and then major increase after PV clamping $(+137 \%[111 ; 164])$. Global HA flow decreased proportionally to hepatectomy percentage, before increasing back with LT. Right QPV increased after left hepatectomy $(+45 \%[42 ; 50])$, and declined with $\mathrm{LT}$, reaching 0 after right PV clamping.

Portal pressures measured after right PV clamping in the 3 cases were respectively of 14,16 and $12 \mathrm{mmHg}$ (Table 2) while the simulated pressures were of $13.1,14.8$ and $11.5 \mathrm{mmHg}$ $(\mathrm{p}=0.25)$. Portocaval gradients measured after right PV clamping in the 3 patients were respectively of 10,11 and $7 \mathrm{mmHg}$ while the simulated gradients were of 9.9, 11.6 and 8.3 $\mathrm{mmHg}(\mathrm{p}=0.5)$. Spearman correlation for the 12 paired values was high ( $\mathrm{r}$ coefficient $=0.94$, $\mathrm{p}=0.02)$.

In order to anticipate the minimal graft weight that should be chosen to avoid the need for portal flow modulation (according to fixed maximal pressure or gradient), we virtually changed the graft weight of each patient and obtained the results presented in Table 1. The minimal graft weight required to avoid PHT with different thresholds proposed for Ppv and portocaval gradients was below the real graft weight in all cases, except for the most restrictive PCG threshold: patient1 was borderline, while patient 2 real size was too low.

\section{DISCUSSION}

On a small series, we succeeded to predict PV pressures and portocaval gradients evolution after RAPID, the differences observed being clinically nonsignificant. This model would have successfully predicted that portal modulation would not have been indicated in these patients bearing liver metastases (non-fibrotic livers). The hemodynamics changes for $\mathrm{Hx}$ are coherent with (15). Compared to the first Hx, small-graft LT reverses Ppv and Qha due to resistance decrease. The right PV-clamping caused the greatest changes. The minimal graft computation showed a sensitivity of the results to each patient and points out the need for clinical consensus about an appropriate threshold.

The main limitations of this work are $1 /$ its small sample size explained by the very preliminary clinical experience reported so far, even by pioneering teams $(1,3) ; 2$ / important assumptions were made for lacking hemodynamic parameters. These statements rely on published cardio-vascular descriptions and our previous experience of 0D models on $\mathrm{Hx}(\mathrm{n}=47$, not reported). For prospective cases we will collect complete data sets.

This preliminary report is promising because it demonstrates that $0 \mathrm{D}$ simulation can be a useful tool for decision-making, thanks to simple input data. In the near future, such a patientspecific model could be of major importance if we transpose the RAPID experience to hepatocellular carcinoma developed on cirrhosis with hyperdynamic circulation, these patients having a high probability of PHT after RAPID since a small graft would even worsen preexisting PHT. In fact, because graft allocation is based on severity of liver failure, mainly depending on MELD (biological gravity) score, and because these patients with hepatocellular 
carcinoma usually have no or minimal liver failure, they have reduced access to liver grafts. The RAPID procedure could allow faster access to transplantation, avoiding high-risk of dropout on list due to graft shortage. This model could also help clinicians for donor selection (decision-making process for minimal liver grafts required) and LT planification (PHT management). Finally, 0D model could lead to expand the RAPID procedure thanks to securing recipients' outcomes and avoiding graft-to-recipient mismatch.

\section{CONCLUSION}

This preliminary report about a very particular LT procedure is the first application of the 0D model to predict liver surgery changes with human data. While requiring further validation on a larger cohort and on fibrotic/cirrhotic organs, it highlights the potential interest to develop innovative tools for better surgical planning. The model could quantify hemodynamic changes of the remnant liver and transplanted graft, highlighting the interaction of the liver with the rest of the circulation. We finally introduced the notion of minimal graft size according to clinically chosen safety thresholds that could allow more patients to undergo LT. 


\section{TABLES}

\begin{tabular}{|c|c|c|c|c|c|c|c|c|c|c|c|c|}
\hline \multicolumn{2}{|c}{$\begin{array}{l}\text { PCG } \\
\text { meas }\end{array}$} & \multicolumn{1}{c}{$\begin{array}{l}\text { PCG } \\
\text { sim }\end{array}$} & $\begin{array}{l}\text { QAo } \\
\text { meas }\end{array}$ & $\begin{array}{l}\text { QAo } \\
\text { sim }\end{array}$ & $\begin{array}{l}\text { PAo } \\
\text { meas }\end{array}$ & $\begin{array}{l}\text { PAo } \\
\text { sim }\end{array}$ & $\begin{array}{l}\text { Ppv } \\
\text { meas }\end{array}$ & $\begin{array}{l}\text { Ppv } \\
\text { sim }\end{array}$ & $\begin{array}{l}\text { Qpv } \\
\text { meas }\end{array}$ & $\begin{array}{l}\text { Qpv } \\
\text { sim }\end{array}$ & $\begin{array}{l}\text { Qha } \\
\text { meas }\end{array}$ & $\begin{array}{l}\text { Qha } \\
\text { sim }\end{array}$ \\
\hline Pat. 1 & 2 & 2,04 & 5 & 5,33 & 80 & 85.70 & 5 & 5.22 & 1 & 1.07 & 0.25 & 0.27 \\
\hline Pat. 2 & 2 & 2.01 & 5 & 5.24 & 81 & 84.22 & 5 & 5.18 & 1 & 1.05 & 0.25 & 0.26 \\
\hline Pat. 3 & 2 & 2.01 & 4.5 & 4.71 & 80 & 84.14 & 5 & 5.18 & 0.9 & 0.95 & 0.22 & 0.24 \\
\hline
\end{tabular}

Table 1: Pre-resection measures and simulations after calibration performed to minimize the L2 score.

QAo : cardiac outpout (L/min) ; Qha: hepatic artery flow (L/min); Qpv: portal vein flow (L/min); meas: measured; PAo : mean arterial pressure ( $\mathrm{mmHg}$ ); PCG: portocaval gradient (mmHg); Ppv: portal vein pressure ( $\mathrm{mmHg})$ : sim : simulated before resection

$$
\text { L2 score }=\sqrt{\sum_{i=1}^{6} w_{i}\left(\left(X_{i}^{\text {meas }}-X_{i}^{\text {sim }}\right) / X_{i}^{\text {meas }}\right)^{2}}
$$

Weight =1 for PCG, QAo, PAo; 2/3 for PPv, 1/3 for QPv and QHa.

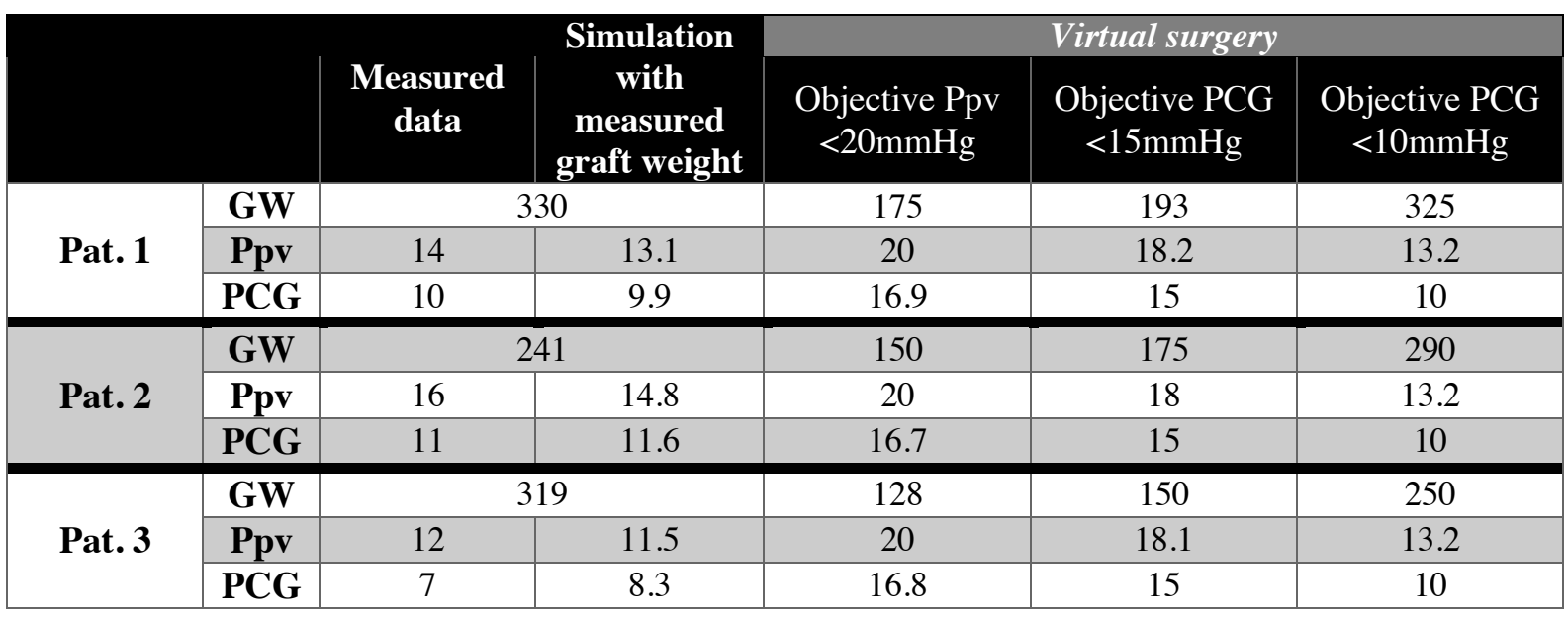

Table 2 : Intraoperative measures (col. 1) after right portal vein clamping, simulation with measured graft weight (col. 2) and simulations from virtual graft weight (col. 3-5). The aim of virtual surgery simulation is to determine the minimal graft weight to respect fixed vascular pressure thresholds.

GW : graft weight (grams); PCG: portocaval gradient (mmHg); Ppv: portal vein pressure (mmHg) 


\section{FIGURES LEGENDS}

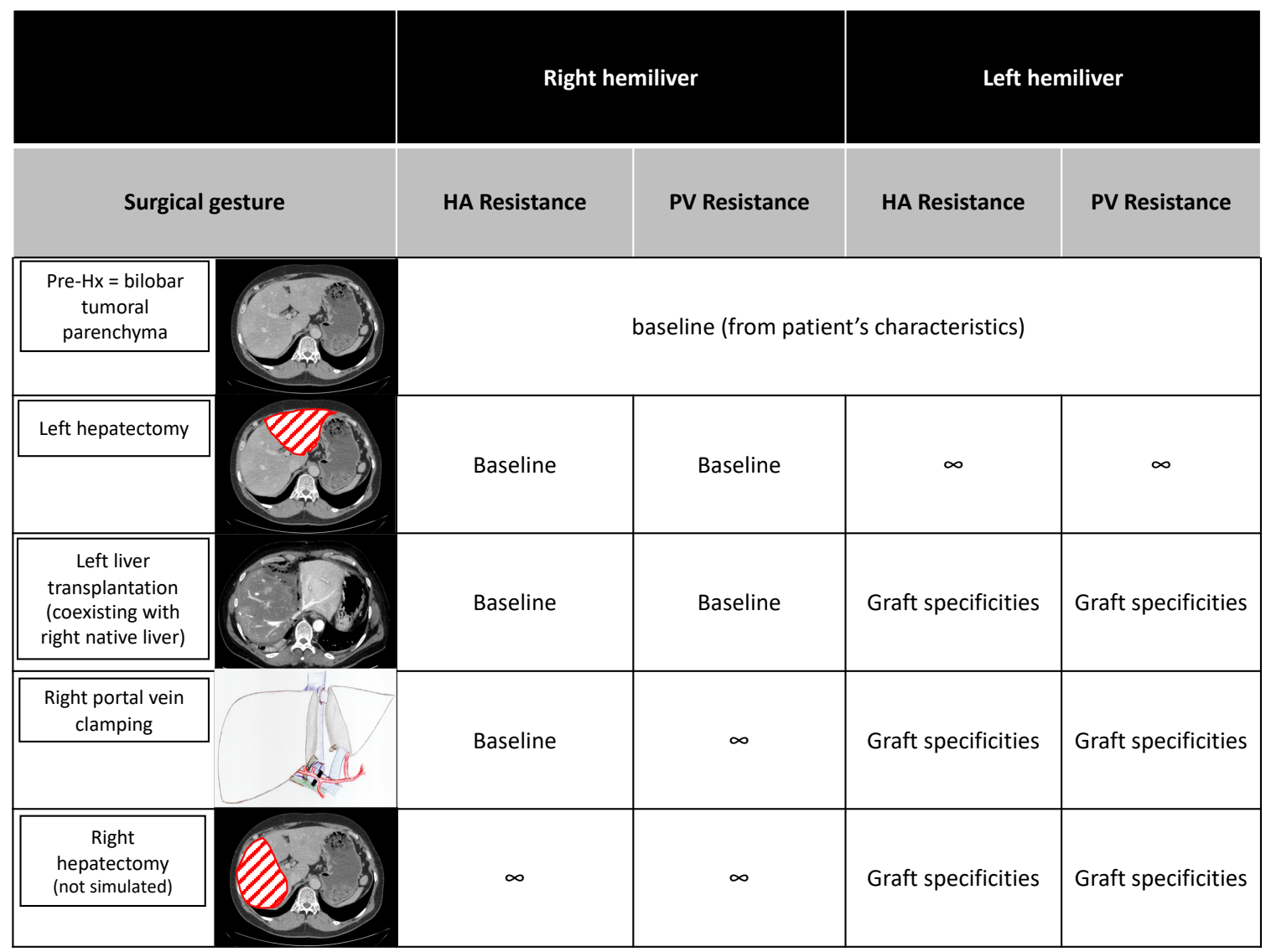

\section{Figure 1}

The successive steps of the sequence are illustrated : partial hepatectomy, transplantation, right portal vein clamping. For each step, modification of the vessels resistance to fit the surgical situation.

HA: hepatic artery; Hx: hepatectomy; LT: liver transplantation; PV: portal vein; $\infty$ : infinite value 


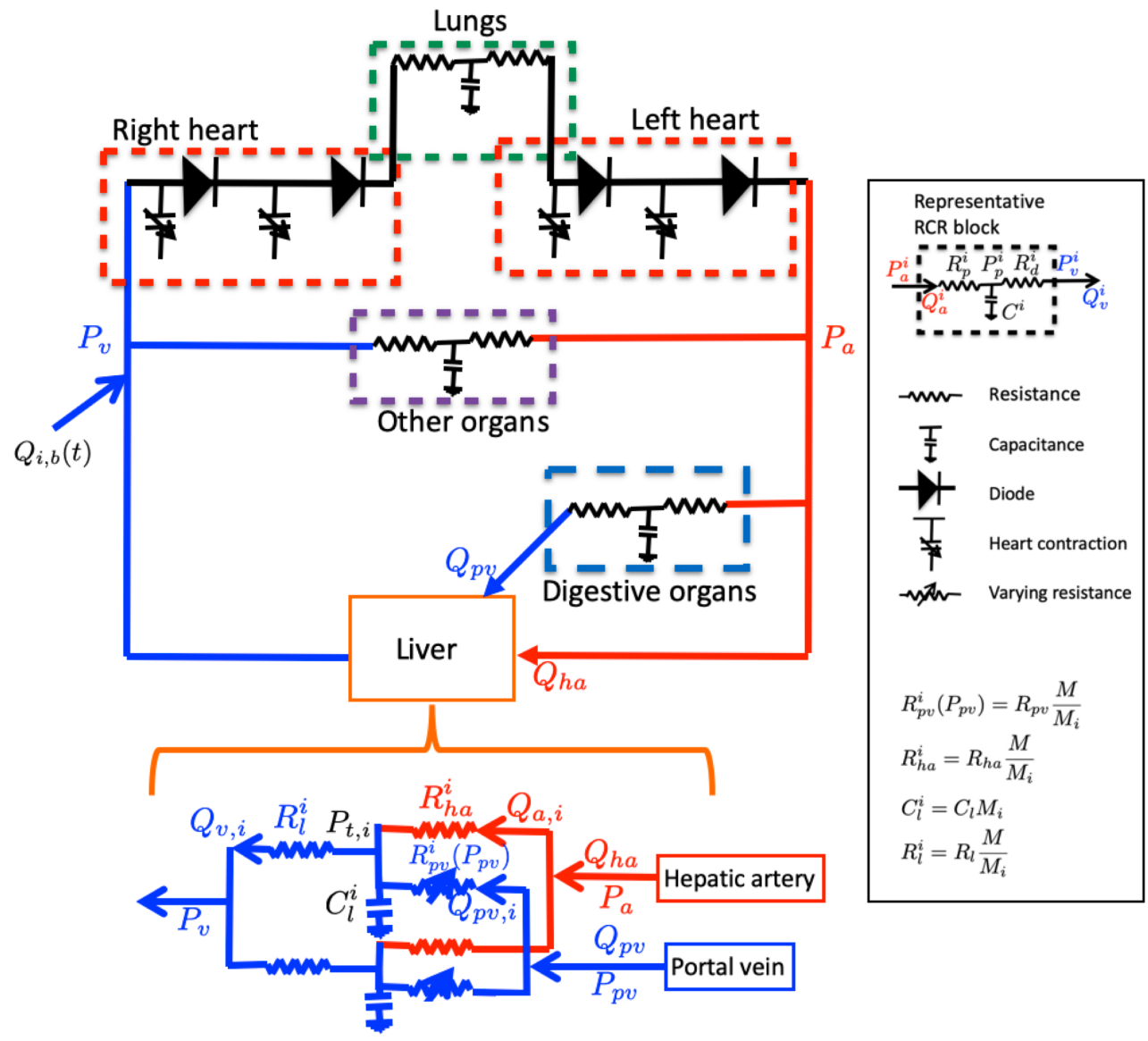

Figure 2

Schematic representation of the 0D closed-loop cardiovascular model adapted for RAPID sequence. Note that the liver is modeled by distinguishing two hemilivers, each one carrying arterial and portal inflows. 

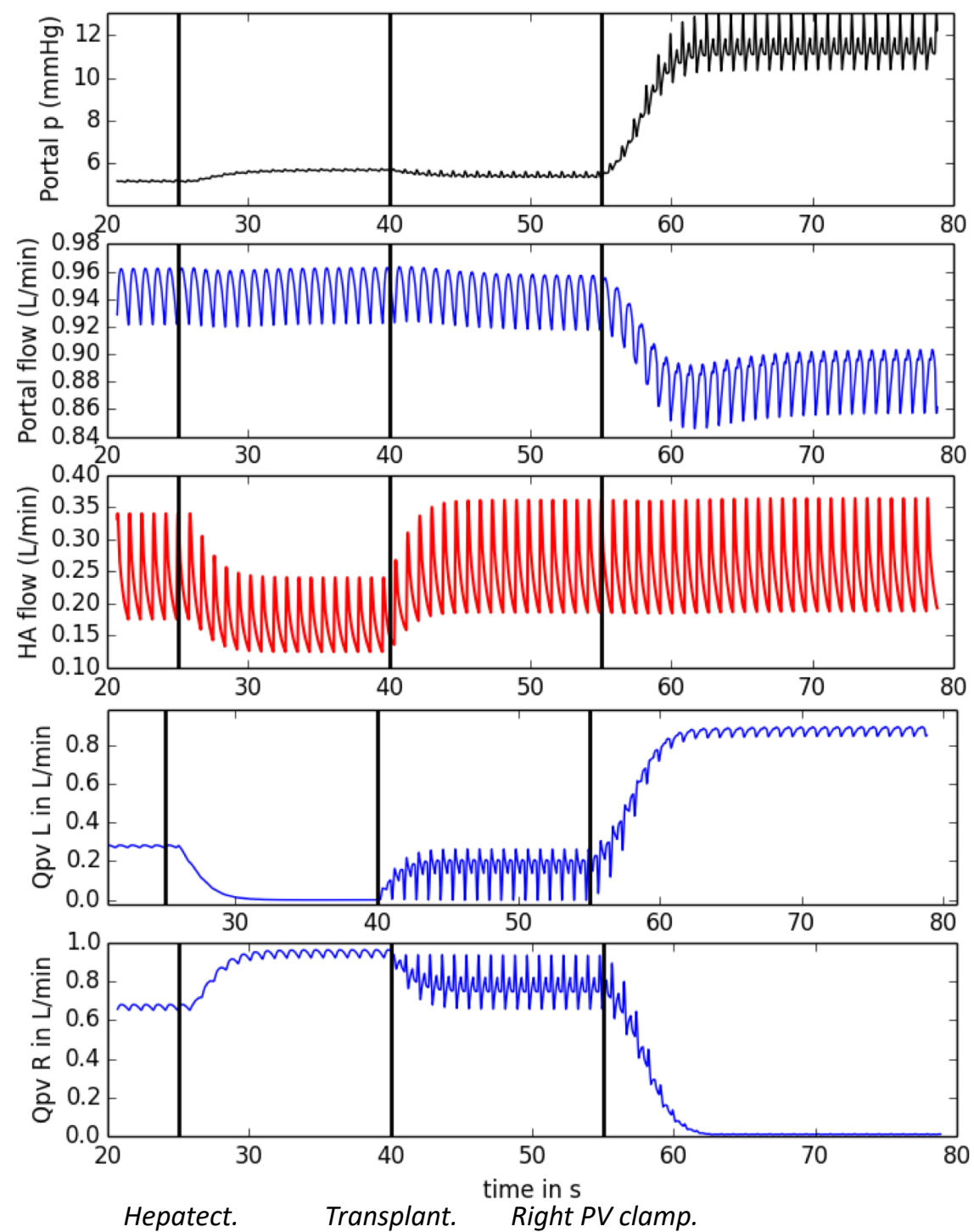

\section{Figure 3}

Example of complete RAPID sequence modeling (excepted delayed final right hepatectomy) for a single patient (hepatectomy at $25 \mathrm{sec}$, transplantation at $40 \mathrm{sec}$, right portal vein clamping at $55 \mathrm{sec}$, end of simulation at $80 \mathrm{sec}$ ).

The beginning of simulation (left side, $20 \mathrm{sec}$ ) starts with the algorithm (pressures, resistances, capacitances) already calibrated with patients' dataset.

$H A$ : hepatic artery; $p$ : pressure; $P V$ : portal vein; $Q_{P V}$ : portal flow for the right $(R)$ and left (L) livers; s: seconds 


\section{REFERENCES}

1. Line P-D, Hagness M, Berstad AE, Foss A, Dueland S. A Novel Concept for Partial Liver Transplantation in Nonresectable Colorectal Liver Metastases: The RAPID Concept. Ann Surg. 2015 Jul;262(1):e5-9.

2. Yagi S, Iida T, Hori T, Taniguchi K, Yamamoto C, Yamagiwa K, et al. Optimal portal venous circulation for liver graft function after living-donor liver transplantation. Transplantation. 2006 Feb 15;81(3):373-8.

3. Königsrainer A, Templin S, Capobianco I, Königsrainer I, Bitzer M, Zender L, et al. Paradigm Shift in the Management of Irresectable Colorectal Liver Metastases: Living Donor Auxiliary Partial Orthotopic Liver Transplantation in Combination With Twostage Hepatectomy (LD-RAPID). Ann Surg. 2018 Jun 18.

4. Wang T, Liang F, Zhou Z, Shi L. A computational model of the hepatic circulation applied to analyze the sensitivity of hepatic venous pressure gradient (HVPG) in liver cirrhosis. J Biomech. 2017 Dec 8;65:23-31.

5. Wang T, Liang F, Zhou Z, Qi X. Global sensitivity analysis of hepatic venous pressure gradient (HVPG) measurement with a stochastic computational model of the hepatic circulation. Comput Biol Med. 2018 Jun 1;97:124-36.

6. Ho H, Sorrell K, Bartlett A, Hunter P. Modeling the hepatic arterial buffer response in the liver. Med Eng Phys. 2013 Aug;35(8):1053-8.

7. Yu HB, Bartlett A, Hunter P, Ho H. Computational simulations for the hepatic arterial buffer response after liver graft transplantation from an adult to a child. Med Eng Phys. 2020 Jan;75:49-52.

8. Debbaut C, Monbaliu DRL, Segers P. Validation and calibration of an electrical analog model of human liver perfusion based on hypothermic machine perfusion experiments. Int J Artif Organs. SAGE PublicationsSage UK: London, England; 2014 Jun;37(6):486-98.

9. Audebert C, Bekheit M, Bucur P, Vibert E, Vignon-Clementel IE. Partial hepatectomy hemodynamics changes: Experimental data explained by closed-loop lumped modeling. J Biomech. 2017 Jan 4;50:202-8.

10. Audebert C, Peeters G, Segers P, Laleman W, Monbaliu D, Korf H, et al. Closed-Loop Lumped Parameter Modeling of Hemodynamics During Cirrhogenesis in Rats. IEEE Trans Biomed Eng. 2018 Oct;65(10):2311-22.

11. Hindmarsh, A. C., Brown, P. N., Grant, K. E., Lee, S. L., Serban, R., Shumaker, D. E., \& Woodward, C. S. (2005). SUNDIALS: Suite of nonlinear and differential/algebraic equation solvers. ACM Transactions on Mathematical Software (TOMS), 31(3), 363396.

12. Garcia D, Pibarot P, Kadem L, Durand L-G. Respective impacts of aortic stenosis and systemic hypertension on left ventricular hypertrophy. J Biomech. 2007;40(5):972-80. 
Published in Journal of Clinical Biomechanics 2020 Vol 77 p 105047

13. Nikolaus Hansen, Sibylle D Mu 1ler, and Petros Koumoutsakos. Reducing the time com- plexity of the derandomized evolution strategy with covariance matrix adaptation (cma-es). Evolutionary computation, 11:1-18, 022003.

14. Vilgrain V, Vullierme M, Djabbari M, Sibert A, Menu Y. [Liver and vascular abnormalities]. J Radiol. 2002 Feb;83(2 Pt 2):255-68.

15. Darnis B, Mohkam K, Schmitt Z, Ledochowski S, Vial J-P, Duperret S, et al. Subtotal hepatectomy in swine for studying small-for-size syndrome and portal inflow modulation: is it reliable? HPB (Oxford). 2015 Oct;17(10):881-8. 